Arroyo González, R. \& Gutierrez Braojos, C. (2016). Competencias escritoras en la formación universitaria del profesorado. Revista Electrónica Interuniversitaria de Formación del Profesorado, 19(3), 135-147.

\title{
Competencias escritoras en la formación universitaria del profesorado
}

Rosario Arroyo González, Calixto Gutierrez Braojos

Universidad de Granada

\section{Resumen}

Este artículo presenta una investigación sobre competencias en la escritura, expresadas por diferentes muestras de estudiantes universitarios. Estas competencias activan procesos universales de desarrollo escritor, esto es: procesos procedimentales-declarativos, condicionales, afectivos y socioculturales. La innovación de esta investigación es describir operativamente las competencias escritoras para su desarrollo en la formación inicial del profesorado. Otra innovación es el estudio de competencias escritoras en diferentes niveles universitarios. Para todo ello se aplica una metodología mixta. Por último, esta investigación fundamenta el diseño e implementación de la enseñanza de competencias escritoras.

\section{Palabras clave}

Estudiantes universitarios; competencias escritoras; análisis estadístico; análisis de contenido

\section{Written competencies in university teacher training}

\begin{abstract}
This paper deals with research in writing competencies, expressed by different samples of universitystudents. These competences activate universal processes of writing development, such as, procedural-declarative, conditional, affective and socio-cultural processes. The innovation
\end{abstract}

Contacto:

Rosario Arroyo González.rarroyo@ugr.es. Universidad de Granada. España 
provided by this paper consists of describe the writing competences in a practical manner for the teachers initial formation. Another new factor included in the paper is the study of writing competences at different university levels. A multimethod methodology was applied during the whole process. Finally this research provides a base for the design and implementation of teaching of writing competences.

\section{Key words}

Higher Education; writing competences; statistical analysis; content analysis

\section{Introducción}

Competencia es la operación, habilidad y/o estrategia inferida que produce una actuación concreta en el futuro y que se evalúa con criterios de eficiencia y oportunidad (Rickheit, Strohner y Vorwerg, 2008). La innovación de este artículo es describir todas las competencias que activan las operaciones escritoras, con criterios de eficiencia profesional y de oportunidad estratégica docente para adaptarse a las exigencias de las sociedades del Siglo XXI. También se trata de averiguar patrones comunes en las competencias expresadas por estudiantes de diferentes niveles universitarios y de diferentes titulaciones en la formación inicial del profesorado. Todo ello con vista a diseñar y aplicar estrategias didácticas que mejoren las competencias escritora del futuro profesional de la educación.

\section{1.- Procesos y competencias escritoras}

Los nuevos retos del Siglo XXI están exigiendo una nueva alfabetización, informacionaldigital, mediática y académica (Guzmán-Simón y García Jimenez , 2014), para el uso de diferentes sistemas lingüísticos, entre los que destaca la práctica social de la escritura. La escritura es también un instrumento de reflexión en la práctica universitaria que permite analizar realidades (Vaquero, Macias y Macazaga, 2014), que conducen a nuevos aprendizajes. Por esto, los procesos de desarrollo escritor es tema de interés recurrente en la investigación desde las últimas décadas del siglo pasado. De todo este bagaje investigador "el epíteto más común, aplicado al acto de la escritura, es que se trata de una tarea compleja" (Myhill y Fisher, 2010, 61)

De la revisión de la investigación sobre la escritura se identifican una serie de competencias, que intervienen en el desarrollo escritor de cualquier lengua y en cualquier contexto comunicativo. Estas competencias son de carácter cognitivos-lingüísticos, metacognivoafectivo y sociocultural (Arroyo, 2006b). Seguidamente se verá como describe la literatura científica las competencias escritoras:

1.- En primer lugar se identifican competencias lingüístico-cognitivas de planificación, transcripción y revisión de la escritura (Alamargot y Fayol, 2009).

2.- En segundo lugar el escritor despliega competencias metacognitivas, tales como poseer una "teoría de las tareas" y una "teoría del texto". Esto supone, por un lado, tener una idea clara y distinta de las posibles finalidades e intenciones del texto que se va a escribir y de cómo éstas condicionan la forma, contenido y estructura del texto y, por otro, poseer un amplio conocimiento metatextual que permita autorregular la escritura (Arroyo, 2009).

3.- La literatura, también destaca las competencias metacognitivas de carácter afectivo. Las más interesantes son la creatividad para aplicar estrategias propias que permite a los 
escritores concentrarse y percibirse competentes en el desarrollo de sus procesos escritores de forma independiente (Fidalgo, Arias-Gundín, García y Torrance, 2010); y la motivación hacia las escritura (Can y Walker, 2011).

4.- Se señalan, además, las competencias socioculturales en la escritura, es decir: competencias sociopolíticas, comunitarias-profesionales y de identidad cultural (Arroyo y Salvador, 2009). En los estudios sociopolíticos de la escritura se comparan el desarrollo de operaciones escritores en escritores de diferentes países (Crossley, WestonMclain y McNamara, 2011). Con respecto a las competencias comunitarias-profesionales, el interés se centra en la diversidad cultural de los estudiantes (Ball, 2006). Por último, en relación a los procesos de desarrollo de la identidad en la escritura, se analiza la expresión de valores culturales en el lenguaje escrito, como fundamento de un currículo educativo que pretende promover la expresión de identidades diferenciadas (Arroyo 2006a; Arroyo y Hunt, 2010).

Sin bien está bastante delimitados todos las competencias escritoras, no existen estudios que describan y evalúen operativamente todas y cada uno de estas competencias para su enseñanza en la formación universitaria. De esto se ocupará este artículo.

\section{2.- Finalidad, objetivos e hipótesis de la investigación}

Los interrogantes que han suscitado esta investigación son los siguientes: ¿los estudiantes, al llegar a la universidad y a su paso por la misma, qué nivel de conciencia tienen sobre las competencias escritoras profesionalizantes? ¿qué operaciones realizan cuando escriben? Por lo tanto, la finalidad de esta investigación es describir, operativamente, las competencias escritoras expresadas por diferentes muestras de estudiantes universitarios, futuros profesionales de la educación. El análisis operativo de las competencias escritoras se realiza a través de la reflexión que el propio estudiante hace de sus actividades cuando escribe. Esta reflexión es incitada a través de una entrevista escrita que desglosa cada competencia escritora en todas las posibles operaciones incluidas en cada competencia.

Para lograr esta finalidad se han perseguido los siguientes objetivos:

1. Describir las operaciones que muestran las competencias escritoras de planificación, transcripción, revisión, de teoría de las tareas, de teoría del texto, de autorregulación, de autocontrol, de motivación, de creatividad, socio-políticas, comunitarias-profesionales y de identidad; en estudiantes universitarios en la Facultad de Ciencias de la Educación.

2. Identificar los diferentes procesos escritores: procedimentales-declarativos, condicionales, afectivos y socioculturales; según el nivel educativo de los sujetos en la Facultad de Ciencias de la Educación

De estos objetivos se derivan la siguiente hipótesis de trabajo:

a) No existen diferencias significativas en la expresión de procesos escritores en función de los niveles educativos universitarios analizados.

\section{3.- Métodología}

En esta investigación se aplicó un diseño de investigación mixto en el que se combina el método de análisis de contenido con la aplicación de estadísticos descriptivos para el logro de los objetivos propuestos y comprobación de la hipótesis. 


\section{1.- Participantes}

La población a quién se dirige ese estudio son los estudiantes de la Facultad de Ciencias de la Educación de la Universidad de Granada. Se obtuvo una muestra de diferentes niveles, aplicando un muestreo no probabilístico deliberado y opinático.

El total de muestra lo compuso 43 estudiantes, de los cuales, 14 eran del tercero y cuarto curso de universidad en las titulaciones de Psicopedagogía y Magisterio y 29 de primer curso de universidad en las titulaciones de Magisterio y Graduado Social

\section{2.- Instrumentos}

Para la recogida de datos se ha aplicado la entrevista de tipo cognitivo, en base a las entrevistas en profundidad diseñadas y aplicadas por Grahan y Harris, (2005) y Salvador y García, (2005); en la que se pregunta al estudiante sobre competencias escritoras. Se trata de una entrevista semiestructurada con preguntas del tipo: "Cuando escribes un texto ¿piensas que el tema, la forma y el idioma en el que se escribe está relacionado con la utilidad que tenga el texto? Pon un ejemplo" (Arroyo, 2009). Esta entrevista incluye 37 preguntas escritas, con un tiempo de realización de una hora, aplicada por un profesor universitario en el primer semestre del curso académico.

\section{3.-Procedimientos}

En este apartado se explicita el proceso de análisis de datos cualitativo y cuantitativo para dar respuesta a los objetivos de este estudio.

Con respecto al análisis de los datos generados tras la aplicación de la mencionada entrevista, se aplicó el Método de Análisis de Contenido. En el proceder de este método fue necesario establecer: a) El universo a analizar: en total 43 entrevistas. b) Las unidades de contexto: la información registrada en cada entrevista. c) Las unidades de registro: la palabra, la frase o el párrafo, siempre que aportase información completa sobre alguna competencia escritora. d) La clasificación de los datos mediante la codificación de cada unidad de registro, usando el Programa Nvivo. e) La elaboración de un sistema de Sistema de Categorías (ver Tabla 1). f) La reducción de los datos mediante el recuento de frecuencia de los códigos que arrojó un total de 3519 codificaciones.

Tabla 1.

Sistema de categorías de procesos, competencias y operaciones escritoras

\begin{tabular}{|c|c|c|}
\hline Procesos & Competencias & Operaciones \\
\hline \multirow{9}{*}{$\begin{array}{l}\text { PROCEDIMEN- } \\
\text { TAL/ } \\
\text { DECLARATIVO }\end{array}$} & \multirow{7}{*}{ Planificación } & Auditorio \\
\hline & & Objetivos \\
\hline & & Génesis de ideas \\
\hline & & Selección de ideas \\
\hline & & Ordenación de ideas \\
\hline & & Fuentes de ideas (dónde busco) \\
\hline & & Registro de ideas \\
\hline & \multirow[b]{2}{*}{ Transcripción } & Sentido gramatical \\
\hline & & Progresión de las ideas y coherencia textual \\
\hline
\end{tabular}




\begin{tabular}{|c|c|c|}
\hline & & Vocabulario \\
\hline & & Selección léxica \\
\hline & & Ortografía y grafía \\
\hline & & Soportes (sobre que escribo) \\
\hline & & Adecuación del texto a lo planificado \\
\hline & Revisión & Modificación de la estructura y léxico de la oración \\
\hline & & Cambios para lograr unidad global y estilo en el texto \\
\hline & & Modificaciones de puntuación, ortografía y grafía \\
\hline & & Revisión por otros \\
\hline & & Revisión por uno mismo \\
\hline CONDICIONAL & Teoría de la tareas & $\begin{array}{l}\text { Funciones socioculturales del texto (para qué escribir } \\
\text { como exigencia social) }\end{array}$ \\
\hline & & $\begin{array}{llll}\text { Características socioculturales y lingüísticas del } \\
\text { auditorio }\end{array}$ \\
\hline & & $\begin{array}{l}\text { Relación entre las funciones y el auditorio; y la forma, el } \\
\text { contenido, la estructura, el código lingüístico y el } \\
\text { soporte del texto }\end{array}$ \\
\hline & & Estrategias para escribir buenos textos (las conozco) \\
\hline & Teoría del texto & $\begin{array}{l}\text { Relación entre el tipo de texto (objetivos, contendido, } \\
\text { forma) y el código lingüístico }\end{array}$ \\
\hline & & $\begin{array}{l}\text { Relación entre el tipo de texto (objetivos, contendido, } \\
\text { estructura, forma) y el tipo de soporte }\end{array}$ \\
\hline & & $\begin{array}{l}\text { Relación entre el tipo de texto (objetivos, contendido, } \\
\text { forma) y el tipo de estructura }\end{array}$ \\
\hline & & $\begin{array}{l}\text { Clasificación de los párrafos y partes del texto según } \\
\text { criterios de jerarquía y unidad }\end{array}$ \\
\hline & Autorregulación & $\begin{array}{l}\text { Selección de estrategias (las elijo) según las } \\
\text { características del texto (objetivos, contendido, forma, } \\
\text { estructura, soporte, código lingüístico) }\end{array}$ \\
\hline & & Auto-instrucciones en el proceso escritor \\
\hline & & Reflexión sobre las propias competencias escritoras \\
\hline AFECTIVO & Autocontrol & $\begin{array}{l}\text { Selección de sentimientos adecuados al tipo texto } \\
\text { (objetivos, contendido, estructura, forma, código } \\
\text { lingüístico y soporte) }\end{array}$ \\
\hline & & Concentración \\
\hline & Motivación & $\begin{array}{l}\text { Recursos humanos y materiales en la escritura (apoyos } \\
\text { en el proceso escritor) }\end{array}$ \\
\hline & & Auto-concepto en las tareas de la escritura \\
\hline & & Expresión de valores propios en la escritura \\
\hline & Creatividad & $\begin{array}{l}\text { Estrategias propias de escritura (estrategias concretas } \\
\text { que aplico ideadas por mi) }\end{array}$ \\
\hline
\end{tabular}




\begin{tabular}{|c|c|c|}
\hline & & Estilos de expresión escrita propios \\
\hline \multirow[t]{9}{*}{$\begin{array}{l}\text { SOCIO- } \\
\text { CULTURAL }\end{array}$} & \multirow[t]{2}{*}{ Sociales-políticas } & $\begin{array}{l}\text { Condicionamientos político-económicos sobre la propia } \\
\text { escritura (soportes, idioma, temas, géneros, utilidad de } \\
\text { mis textos) }\end{array}$ \\
\hline & & $\begin{array}{l}\text { Funciones sociales de la propia escritura (mis escritos } \\
\text { mejoran la sociedad) }\end{array}$ \\
\hline & \multirow{4}{*}{$\begin{array}{l}\text { Comunitarias- } \\
\text { profesionales }\end{array}$} & $\begin{array}{l}\text { Usos cotidianos y/o profesionales de la escritura } \\
\text { (cuándo y dónde escribo) }\end{array}$ \\
\hline & & $\begin{array}{l}\text { Intereses comunitarios y/o profesionales en los usos } \\
\text { cotidianos de la propia escritura (mis escritos sirven } \\
\text { para conocer o ayudar a los demás) }\end{array}$ \\
\hline & & $\begin{array}{l}\text { Códigos lingüísticos utilizadas en los usos cotidianos de } \\
\text { la propia escritura }\end{array}$ \\
\hline & & $\begin{array}{l}\text { Estrategias colaborativas en la construcción de los } \\
\text { propios textos }\end{array}$ \\
\hline & \multirow{3}{*}{ Identidad } & Experiencias o ideas expresadas en los propios escritos \\
\hline & & Domino de la lengua escrita materna \\
\hline & & $\begin{array}{l}\text { Intereses propios en los uso de la escritura (mis escritos } \\
\text { me sirven a mi) }\end{array}$ \\
\hline
\end{tabular}

El procedimiento básico usado para garantizar la fiabilidad es "la triangulación". Una estrategia de triangulación aplicada en esta investigación es la "triangulación de fuentes de datos", utilizando sujetos diferentes titulaciones, que reflejan la variedad de estudiantes matriculados en la Facultad de Ciencias de la Educación de la Universidad de Granada. Otras estrategias de triangulación, aplicada, es la "triangulación de investigadores", en sesiones de discusión sobre las unidades de registro donde no existían coincidencias de codificación para llegar a un consenso.

La validez del sistema de categorías se estableció aplicando la vía inductiva y la vía deductiva. En una investigación previa (Arroyo, 2013) la vía inductiva había permitido identificar y clasificar los procesos escritores expresado por los sujetos de una muestra, en categorías, subcategorías y operaciones (ver Tabla 2), aplicando el Programa Nvivo. Posteriormente, por la vía deductiva el sistema de categorías y la entrevista cognitiva fueron sometidos al Juicio de Expertos. Para ello se aplicó a los expertos un cuestionario que valoraba los instrumentos mencionados. Con el propósito de analizar el grado de acuerdo entre los jueces expertos se aplicó W de Kendall y se calculó el coeficiente de correlación intraclase. Los resultados obtenidos demostraron que las valoraciones de los jueces expertos en todas las dimensiones analizadas, fue positiva (Arroyo y GutiérrezBraojos, 2013).

\section{4.-Resultados}

En la Tabla 2 se presentan los recuentos de frecuencias para cada proceso (categoría) y competencia (subcategoría) escritor, expresados por los sujetos de la muestra, clasificados por niveles universitarios y titulación. También se presentan los porcentajes de frecuencias referidos al total de codificaciones de cada Nivel/Titulación. 
En líneas generales, los resultados del análisis indican la expresión de procesos y competencias escritoras son muy similares entre las diferentes titulaciones universitarias docentes.

Tabla 2.

Tabla de frecuencias de procesos y competencias escritoras

\begin{tabular}{|c|c|c|c|c|c|c|c|c|c|c|c|c|c|c|c|c|c|}
\hline Nivel/Títulación & Sp & St & $\mathrm{Sr}$ & SD & Sta & Stx & Sarr & SC & Sac & Sm & Sc & SA & Ss & Scp & Si & SSC & ST \\
\hline & 87 & 62 & 63 & 212 & 31 & 25 & 23 & 79 & 1 & 56 & 27 & 84 & 11 & 62 & 35 & 108 & 483 \\
\hline \multirow[t]{2}{*}{$4^{\circ} \mathrm{PS}(n=5)$} & $18 \%$ & $13 \%$ & $13 \%$ & $44 \%$ & $6 \%$ & $5 \%$ & $5 \%$ & $16 \%$ & $0 \%$ & $11 \%$ & $6 \%$ & $17 \%$ & $2 \%$ & $13 \%$ & $8 \%$ & $23 \%$ & $100 \%$ \\
\hline & 368 & 296 & 118 & 782 & 79 & 96 & 115 & 290 & 1 & 187 & 81 & 269 & 40 & 167 & 127 & 334 & 1675 \\
\hline \multirow[t]{2}{*}{$1^{\circ} / \operatorname{LE}(n=20)$} & $22 \%$ & $18 \%$ & $7 \%$ & $47 \%$ & $5 \%$ & $6 \%$ & $7 \%$ & $18 \%$ & $0 \%$ & $11 \%$ & $5 \%$ & $16 \%$ & $2 \%$ & $10 \%$ & $7 \%$ & $19 \%$ & $100 \%$ \\
\hline & 169 & 100 & 69 & 338 & 43 & 29 & 73 & 145 & 3 & 89 & 51 & 143 & 14 & 72 & 50 & 136 & 762 \\
\hline \multirow[t]{2}{*}{$3 \% / P(n=8)$} & $22 \%$ & $13 \%$ & $9 \%$ & $44 \%$ & $6 \%$ & $4 \%$ & $10 \%$ & $20 \%$ & $0 \%$ & $12 \%$ & $7 \%$ & $19 \%$ & $2 \%$ & $9 \%$ & $6 \%$ & $17 \%$ & $100 \%$ \\
\hline & 141 & 95 & 34 & 270 & 25 & 18 & 41 & 84 & 2 & 60 & 25 & 87 & 14 & 81 & 63 & 158 & 599 \\
\hline $1^{\circ} / \mathrm{ES}(n=7)$ & $24 \%$ & $16 \%$ & $6 \%$ & $46 \%$ & $4 \%$ & $3 \%$ & $7 \%$ & $14 \%$ & $0 \%$ & $10 \%$ & $4 \%$ & $14 \%$ & $2 \%$ & $14 \%$ & $10 \%$ & $26 \%$ & $100 \%$ \\
\hline
\end{tabular}

Nota1: “PS”, 4\% Psicopedagogía; “LE”, 1\%/ Maestro; “P”, 3 Maestro; “ES”, $1^{\circ}$ de Educación Social

Nota 2: S:sumatoria de frecuencias

Competencias: Sp: planificación, St: transcripción, Sr: revisión; Proceso: SD: Procedimental-declarativo

Competencias: Sta: teoría de la tarea, Stx: teoría del texto, Sarr: autorregulación; Proceso: SC: Condicional

Competencias: Sac: autocontrol, Sm: motivación, Sc: creatividad; Proceso: SA: Afectivo

Competencias: Ss: socio-políticas, Scp: comunitarias-profesionales, Si: identidad; Proceso: SSC: Sociocultural

ST: suma total de frecuencias por Nivel/Titulación

Seguidamente se describirán las operaciones escritoras que realizan los estudiantes entrevistados y que describen las diferentes competencias escritoras. Para ello se extraerán citas textuales de las entrevistas y se pondrá en paréntesis el código de la Titulación y el número de la entrevista (ejemplo: PS01= Titulación de Psicopedagogía, Entrevista 01).

4.1.- Competencias de planificación, transcripción y revisión: proceso procedimentaldeclarativo de la escritura

En todas las titulaciones se obtienen frecuencias entre el $18 \%$ y el $24 \%$ en la competencias de planificación (Sp), expresadas en operaciones tales como: a) registrar idea: "escribirlas en un papel" (PS01); b) búsqueda de fuentes de ideas: "en internet (google..) y distintas enciclopedias" (LE08) “... en revistas" (LE15); C) ordenar ideas: "hago un pequeño esquema de ideas sobre las que quiero poner en el texto" (LE22); d) plantearse objetivos "antes de escribir un texto pienso para que y porqué estoy escribiendo"(LE22); e) seleccionar de ideas: "Anoto ideas que pienso que me van a ser útiles para desarrollar alguna parte del texto o conclusiones que me surgen" (P31); $f$ ) generar ideas: "antes de escribir el texto pienso en el tema del texto" (ES38); g) considerar el auditorio: "ante todo que el mensaje que quieres transmitir llegue a los destinatarios, un profesor, un amigo, tu pareja..." (ES39).

La transcripción de la escritura, entre el $13 \%$ y el 16\%, (St) se expresa con operaciones, tales como: a) sentido gramatical: "intento escribir las frases completas" (ES42); b) progresión de ideas y coherencia textual: "utilizo nexos como 'por consiguiente", siguiendo en esa línea al igual que en la anterior" (ES41); c) vocabulario: "intento buscar sinónimos de los que conozco" (P35); d) selección léxica: "a veces me gustan palabras que leo en algún sitio o que 
alguien usa..." (Р30); e) ortografía y grafía: "Punto y aparte....pongo coma, e intento no cometer faltas" (P23), "aplico normas de acentuación, sangría, párrafos..." (P28); f) selección de soportes: "Papel, bolígrafo, folios para escribir..." (LE22) "procesadores de textos como Microsoft Word" (LE15).

Por último, con frecuencias entre el $6 \%$ y el 13\%, la competencia de revisión ( $\mathrm{Sr}$ ) es expresada por los sujetos de la muestra en operaciones del tipo: a) adecuación del texto a lo planificado: "después de escribir cambio palabras, frases, párrafos" (LE11); b) modificación de la estructura y léxico de la oración: "después de escribir un texto quito y cambio nexos" (LE10), "después de escribir un texto añado palabras, frases, nexos" (LE10); c) cambios para lograr la unidad global y estilo del texto: “... las voy relacionando, unas con otras [las ideas] hasta que decido como quiero que vaya quedando el texto... cuando me doy cuenta de que alguna idea está siendo redundante modifico o cambio alguna parte..." (PSo4); d) modificación de puntuación, ortografía y grafía: "después de escribir un texto añado o quito signos de puntuación y cambio la caligrafía” (PSO3); e) revisión por otros o por uno mismo: "para saber que tengo que modificar en el texto pues leerlo como si estuviese leyendo para la clase" (LE08)

En definitiva es un patrón común de toda las muestraque se obtengan valores bastantes homogéneos entre el $44 \%$ y $47 \%$, en el proceso procedimental-declarativo (SD).

4.2.-Competencias socio-políticas, comunitarias-profesionales y de identidad: proceso sociocultural de la escritura

El proceso sociocultural (SSC) presenta una oscilación de frecuencias entre el $17 \%$ y el $26 \%$.

La competencia comunitarias-profesional de la escritura (Scp), con frecuencias entre $9 \%$ y el $14 \%$, se expresa en las operaciones siguientes: a) usos cotidianos y profesionales de la escritura: "escribo fundamentalmente en el ámbito académico" (PSO3), "escribo en mi casa por mi cuenta" (LE06), b) intereses comunitario y/o profesionales en los usos cotidianos de la escritura: "las cartas con mis amigas sirven para conocerlas mejor" (LE07); c) códigos lingüísticos utilizados en los usos cotidianos de la propia escritura: "según la utilidad del texto utilizo figuras retóricas, lenguaje específico y técnico" (ES36); d) estrategias colaborativas en la construcción del texto: "hago textos colaborativos en los trabajos de clase" (ES38).

La competencia de identidad en la escritura (Si), con frecuencias entre el $6 \%$ y el $10 \%$, es descrita en operaciones del tipo: a) experiencias e ideas expresadas en los propios escritos: "escribo relatos breves, para ello me inspiro en documentales... tengo un relato acerca de un homosexual..." (P30); b) dominio de lengua escrita materna: "mis padres me ayudaron a escribir" (P29), e) intereses propios en el uso de la escritura: "mis escritos han servido para que mi escritura sea lo más correcta posible" (LE25), "mis textos han mejorado mi escritura y mi vida" (PSO2).

Se puede comprobar que las frecuencias en la competencia sociopolítica de la escritura (Ss) caen por debajo del $5 \%$. Esta competencia se refieren a la toma de conciencia de los condicionamientos, políticos y económicos que determinan los soportes, los idiomas, los temas, los géneros y las finalidades de los textos propios. La competencia sociopolíticas también se refiere a la toma de conciencia sobre la utilidad social de los propios textos, expresadas de la siguiente forma: "Pienso que lo que he escrito ha servido para algo o para alguien" (ES34).

En definitiva es un patrón común de toda la muestra es que se obtengan menor frecuencia (entre el $17 \%$ y el $26 \%$ ) en el proceso sociocultural (SSC), que en el proceso procedimentaldeclarativo (SD) de la propia escritura, con frecuencias, este último, entre el $44 \%$ y el $47 \%$. 
4.3.-Competencias de teoría de la tarea, de teoría del texto, de autorregulación, de autocontrol, de motivación y de creatividad: proceso condicional y afectivo de la escritura

$\mathrm{Si}$ se observan los porcentajes de frecuencias de las competencias en el proceso condicional, destaca la competencia de autorregulación (Sarr), con una frecuencia entre el $5 \%$ y el 7\%; descrita en operaciones tales como: a) selección de estrategias según las características del texto: "en los textos que escribo varío las estrategias y el código lingüístico" (ES40); b) Auto-instrucciones en el proceso escritor: "cuando escribo un texto me digo a mi mismo lo que tengo que hacer para escribir un buen texto" (ES36); c) reflexión sobre las propias competencias escritoras: "para mi es importante saber donde se tiene que escribir una pausa y cómo se escriben las palabras...si voy a escribir un texto argumentativo se que tiene que tener..." (LE14). Dentro del proceso condicional le siguen, en porcentajes de frecuencias, las competencias de teoría de las tareas (Sta) y de teoría del texto (Ttx). Se observan frecuencias algo más bajas en la teoría de las tareas con respecto a la teoría del texto. Las competencias de teoría del texto se expresan en operaciones que muestran un conocimiento de la relación entre el tipo de texto y el código lingüístico, el soporte y la estructura del texto: "mi texto es diferente según los aparatos" (LEog), "por ejemplo si escribo un texto argumentativo deberá tener las partes de las que consta" (LE16). También la teoría del texto (Stx) se muestra en la operación de clasificar párrafos y partes del texto según criterios de jerarquía y unidad. Esta operación ha sido expresada, parcialmente, solo en una entrevista: "... al final suelo necesitar una conclusión que permita dejar al lector con una buena idea final de lo que ha leído" (LE17).

Por otra parte, poseer una teoría de la tarea (Sta), con una frecuencia entre el $4 \%$ y el $6 \%$, es una competencia que se muestra en operaciones tales como: conocer las funciones socioculturales del texto, las características socioculturales y lingüísticas del auditorio y las relaciones de esto con el tipo de texto. Estas operaciones se expresan de modo parcial del siguiente modo: "Para quién esté dirigido, y su utilidad puede ser diferente dependiendo del texto pero puede servirle como apoyo para escribir textos relacionados con el tema" (LE22). Otra operación de la competencia que presume una teoría de las tareas es conocer estrategias para escribir buenos textos: "cuando escribo un texto sé lo que necesito y lo que tengo que hacer para lograr terminarlo" (P29).

En lo que se refiere al proceso afectivo destaca el hecho de que los mayores porcentajes de frecuencias (entre el 10\% y 12\%) se acumulen en la competencia de motivación (Sm) hacia la escritura. Esta competencia se muestra en la disposición de apoyos humanos y materiales en la escritura y en el auto-concepto en las tareas escritoras, operaciones que se expresan del siguiente modo: "busco en internet la mejor manera de hacerlo...o pregunto a compañeros" (LE08), "he mejorado mi caligrafía, mis faltas de ortografía y mi expresión" (LE10). Es de destacar que la mayor frecuencia en la competencia de motivación corresponde a la expresión de apoyos en la escritura.

En este proceso afectivo las frecuencias proporcionales descienden entre el $4 \%$ y el $6 \%$, en la competencia creativa (Scp). Esta competencia se muestra en operaciones de expresión de valores con la escritura, en el uso de estrategias propias y en la aplicación de un estilo personal en la escritura. Estas operaciones se expresan del siguiente modo: "reflexionamos sobre valores que nos transmite tal o cual pasaje..."(P30), " tener las ideas claras, organizar estas ideas y darles forma a través de la escritura; además utilizaré toda las fuentes de información posibles a mi alcance sobre el tema" (PS05). Por último, destacar la nula frecuencia proporcional de expresión de la competencia de autocontrol (Sac) en la escritura, en todas las titulaciones. 


\section{4.-Competencias escritoras expresadas por los sujetos de los diferentes niveles} universitarios

Debido a una ausencia de normalidad en determinadas variables, y varianzas no homogéneas, se optó por aplicar la U de Mann-Withney para el contraste de los procesos escritores entre ambos grupos. El primero con estudiantes de primer curso y el segundo con estudiantes de tercer y cuarto curso, para determinar algún avance en los procesos escritores.

Con la aplicación de la $U$ de Mann-Whitney, los estadísticos de contrastes y el p-valor asociado indican nulidad de diferencias significativas entre ambos grupos en todos los procesos escritores analizados: procedimentales-declarativos, condicionales, afectivos y socioculturales (ver Tabla 3). Finalmente, se calculó el tamaño del efecto (Cohen, 1988). Los resultados obtenidos indicaron una limitada varianza en los procesos escritores.

Tabla 3.

Comparación entre grupos aplicando U de Mann_Whitney

\begin{tabular}{|l|c|c|c|c|c|c|}
\hline \multirow{2}{*}{$\begin{array}{l}\text { Variables } \\
\text { dependientes }\end{array}$} & $\begin{array}{c}\text { Grupos de Primer } \\
\text { año } \\
(n=29)\end{array}$ & $\begin{array}{c}\text { Grupos de } 3^{\circ} \text { y } 4^{\circ} \text { año } \\
(n=14)\end{array}$ & \multicolumn{3}{|c|}{ Valores estadísticos } \\
\cline { 2 - 7 } & $R^{\circ}$ & $R^{\circ}$ & $U$ & $z$ & $p$ & $d$ \\
\hline $\begin{array}{l}\text { Proceso } \\
\text { procediemtal- } \\
\text { declarativos }\end{array}$ & 22.19 & 21.61 & 195.5 & -.14 & .87 & .02 \\
\hline Proceso condicional & 21.95 & 22.11 & 201.5 & -.04 & .97 & .01 \\
\hline Proceso afectivo & 21.78 & 22.46 & 196.5 & -.17 & .87 & .03 \\
\hline Proceso sociocultural & 21.88 & 22.85 & 199.5 & -.09 & .93 & .01 \\
\hline Total & 22.09 & 21.82 & 200.5 & -.07 & .95 & .01 \\
\hline
\end{tabular}

Por tanto, se puede interpretar que no se observa un avance en el uso de los procesos escritores en los estudiantes de segundo y tercer curso con respecto a los de primer curso.

\section{5.- Discusión y Conclusiones}

Seguidamente se presentan las conclusiones de esta investigación en relación a los objetivos e hipótesis.

Con respecto a las similitudes en competencias escritoras expresadas por los sujetos de la muestra, se descubren patrones comunes, estos son:

- El proceso procedimental-declarativo obtiene las frecuencias más altas (con valores entre $44 \%$ y $47 \%$ ) y, además, se expresan competencias de planificación, transcripción y revisión de la escritura. Estos resultados coinciden con una investigación previa (Arroyo, 2013) realizados con estudiantes universitarios.

- Sigue el proceso sociocultural, en cuanto a tendencia a la baja (frecuencias entre el $16 \%$ y el $27 \%$ ). Destaca, en esta tendencia, la operación de la toma de conciencia de los condicionamientos, políticos y económicos (competencia sociopolítica). Esto 
significa el desconocimiento de la influencia político-económico en la propia escritura por parte de los estudiantes entrevistado, lo que supone escasa conciencia crítica en el lenguaje escrito. En este sentido Castello y Donahue (2012), plantean la necesidad de actividades académicas escritas en la universidad que despierten el pensamiento crítico.

- Con respecto a los procesos condicionales y los procesos afectivos la tendencia común es la oscilación de frecuencias con tendencia, igualmente, hacia la baja (entre $14 \%$ y el $20 \%$ y entre el $14 \%$ y el $19 \%$, respectivamente). Destaca la presencia de la competencia de autorregulación del proceso condicional de la escritura. Este hallazgo coincide con otras investigaciones que demuestran la presencia de esta competencias a medida que aumenta la edad (García y Fidalgo, 2003).

- En lo que se refiere al proceso afectivo destaca la competencia de motivación hacia la escritura. Esta competencia se sustenta, sobre todo, en la disposición de apoyos materiales en la escritura por encima de un auto-concepto positivo hacia la misma.

Por último, se puede afirmar que se cumple la hipótesis planteada, puesto que no se observa un avance en la expresión procesos escritores en los estudiantes de segundo y tercer curso con respecto a los de primer curso, lo que, de alguna forma, apunta a que el paso por la Universidad no es un variable influyente en la expresión de competencias escritoras. Coincidiendo esto con los hallazgos de Dysthe, (2007) en sus estudio con Universidades Noruegas, donde se demuestra que en la práctica regular de la escritura en la universidad no es suficiente para mejorar la escritura de los estudiantes y, por lo tanto, es necesario organizar la enseñanza académica en la escritura en los niveles universitarios.

En definitiva, se puede concluir que en base a una evaluación diagnóstica de la escritura, cuyo criterio de evaluación han sido todas las competencias escritoras para el desarrollo profesional, incluidas en el sistema de categorías presentado; esta investigación pone de manifiesto importantes carencias en competencias escritoras de estudiantes universitarios, tales como: auto-control, creatividad, teoría del texto, teoría de la tarea, identidad y competencias socio-políticas. En esta misma línea un estudio de Guzmán-Simón y García Jiménez, (2014), demuestra que los estudiantes universitario hacen solo un uso instrumental de la escritura, es decir, escriben solo por demandas académicas y en papel; lo que puede está impidiendo el despliegue de competencias escritoras de alto nivel detectado en este estudio.

Por consiguiente, se están diseñando, aplicando y evaluando programas educativos interactivos para futuros docentes y universitarios, en general. Estos programas promueven, tanto el dominio de competencias de autorregulación de la escritura; como el aprendizaje de estrategias de revisión de la escritura: por ejemplo las rúbricas (Andrade, Wang, Du y Akawi, 2009); y de estrategias de estructuración metatextual que incrementen la percepción de autoeficacia escritora (Pajares y Valiente, 2006). Por último estos programas se centran en la reflexión individual y colaborativa sobre los condicionamientos socioculturales de la propia escritura y sobre su propio potencial creativo utilizando diversas lenguas y plataformas de formación on-line (Arroyo, 2012)

\section{Referencias}

Alamargot, D., y Fayol, M. (2009). Modelling the Development of Written Composition. En R. Bear, D. Myhill, M. Nystrand y J. Riley (Eds), The Sage Handbook of Writing Development (pp:23-47). London: Sage. 
Andrade, H.L., Wang, X., DU, Y. y Akawi. R. L. (2009). Rubric-Referenced Self-Assessment and Self-Efficacy for Writing. The Journal of Educational Research, 102(4), 287-300.

Arroyo, R. (2006a). La interculturalidad en los procesos de la composición escrita. Investigación en la escuela, 59, 91-102.

Arroyo, R. (2006b). Desarrollo de la escritura en un contexto multicultural. Una investigación etnográfica. Revista de Investigación Educativa, 24 (2), 305-328.

Arroyo, R. (2009). Desarrollo Metacognitivo y Sociocultural de la Composición Escrita. Interculturalidad y Tecnologías en la enseñanza de la escritura multilingüe. Granada: Nativola.

Arroyo, R. (2012). Gestión de Programas Interculturales. En M. Lorenzo y M. López (Coors.), Respuestas Emergentes desde la organización de instituciones educativas (pp.225-228). Granada: Universidad de Granada.

Arroyo, R. (2013). Descripción de procesos en la composición escrita de estudiantes universitarios para un desarrollo multilingüe y tecnológico. Revista de Investigación Educativa, 31 (1), 167-184.

Arroyo, R. y Gutiérrez-Braojos, C. (2013). Validación de una entrevista metasociocognitiva y un sistema de categorías, para la investigación multilingüe de procesos escritores. Manuscrito presentado para su publicación.

Arroyo, R. y Hunt, C.I. (2010). Strategies, Tools and Techniques for the Development of Written Communication Metasociocognitive Processes. En N. Mertens (Ed.), Writing Processes, Tools and Techniques (pp.57-74). New York: Nova Science Publihers.

Arroyo, R. y Salvador, F. (2009). Research on cognitive, social and cultural processes of written communication. Cognitive Processing, 10 (3), 263-268.

Ball, A. (2006). Teaching Writing in Culturally Diverse Classrooms. En McA. Charles, G. Steve y F. Jill. (Eds), Handbook of Writing Research (pp.293-310). New York: Guilford Press.

Can, G. y Walker, A. (2011). A Model for Doctoral Students' Perceptions and Attitudes toward Written Feedback for Academic Writing. Research in Higher Education, 52(5), 508-536.

Castelló, M. y Donahue, C. (eds.). (2012). University writing: Selves and tets in academic societies. Bingley (UK): Emerald.

Crossley, S.A., Weston, J.L., Mclain, S., Susan T.y Mcnamara, D. (2011). The Development of Writing Proficiency as a Function of Grade Level: A Linguistic Analysis. Written Communication, 28 (3), 282-311.

Dysthe, O. (2007). How a Reform Affects Writing in Higher Education. Studies in Higher Education, 32 (2), 237-252.

Fidalgo, R., Arias-Gundín, O., García, N. y Torrance, M. (2010). Cognitive Strategic and Selfregulated Instruction in Writing Processes. En N. Mertens (Ed.), Writing Processes, Tools and Techniques (pp.129-152). New York: Nova Science Publishers.

García J.N. y Fidalgo, R. (2003). "Cambios en la metacognición de los procesos psicológicos de la escritura en estudiantes de $3^{\mathrm{a}}$ EP a $3^{\mathrm{a}}$ ESO". Revista de Psicología General y Aplicada, 2(56), 239-251.

Graham, S. y Harris, K. (2005). Writing Better: Strategies for Teaching Students with Learning Difficulties. Baltimore: Brookes Publising. 
Guzmán-Simón, F. y García-Jiménez, E. (2014). Los hábitos lecto-escritores en los alumnos universitarios. Revista Electrónica Interuniversitaria de Formación del Profesorado, 17 (3), 79-92.

Myhill, D. y Fisher, R. (2010). "Writing development: cognitive, sociocultural, linguistic perspectives". Journal of Research in Reading, 33 (1), 1-3.

Pajares, F. y Valiente, G. (2006). Self-efficacy beliefs and motivation in writing development. En C. MacArthur, S. Graham y J. Fitzgerald (Eds), Handbook of Writing Research (pp. 158-170). New York: Guilford Press.

Rickheit, G.; Strohner, H. y Vorwerg, C. (2008). "The concept of communicative competence". En: Rickheit y Strohner (comp.). Handbook of Communication Competence. Berlin: Mouton de Gruyter, 15-62.

Salvador, F. y García A. (2005). Metodología de la Investigación. En F. Salvador, (Ed.). La expresión escrita de alumnos con necesidades educativas especiales. Procesos cognitivos (pp. 45-70). Archidona: Aljibe.

Vaquero Barba, A.; Macias Muñoz, O. y Macazaga López, A. (2014). La práctica corporal y la imagen corporal: reconstruyendo significados. Revista Electrónica Interuniversitaria de Formación del Profesorado, 17 (1), 163-179. 